\title{
Dermatitis artefacta: a case report
}

\author{
Seshadri Chatterjee, Kaberi Bhattacharya
}

\section{Summary}

Dermatitis artefacta is rarely encountered and has a variable dermatological presentation. It is also a diagnosis of exclusion, thereby posing a challenge to the clinician. We report a case of dermatitis artefacta associated with depressive disorder which responded to management with anti-depressants and supportive psychotherapy.

\section{Introduction}

Psychocutaneous disorders include a vast spectrum of dermatological diseases that are somatic manifestations of disordered thinking, behaviour, or perception. Although a relationship between certain dermatological disorders and the psychological state of patients has been well recognised, there is a lack of large-scale studies on these disorders due to their rarity. Among them, dermatitis artefacta poses a particular challenge to the clinician because the pattern of the skin lesions can be extremely variable depending on how it is caused, its defining feature being the inability to attribute the lesion to a known medical condition.

\section{Case Report}

We report a case of dermatitis artefacta which highlight the condition's variable presentation, its diagnostic indicators and complex management issues. A 20 -yearold female presented with multiple skin lesions distributed over accessible sites of the body, mostly on the face. They had tapering ends and were in various stages of healing with little inflammation.

Multiple, well-demarcated lesions on the whole of the face and the dorsum of both forearms were noted, aligned longitudinally. The lesions had tapering ends and were in various stages of healing. Their appearance did not correspond to known dermatological conditions. As wounds inflicted by another individual were a consideration, a detailed dermatological survey was conducted. It revealed that the lesions were confined to easily accessible areas with sparing of less accessible areas such as the back (Figure 1).

On inquiry, the patient reported that the lesions had appeared seven days previously. She denied any knowledge of the origin, cause, or circumstances in which these skin lesions appeared or progressed; neither did she admit self-infliction. consideration, a detailed dermatological survey was conducted. It revealed that the lesions were confined to easily accessible areas with sparing of less accessible areas such as the back (Figure 1).

On inquiry, the patient reported that the lesions had appeared seven days previously. She denied any knowledge of the origin, cause, or circumstances in which these skin lesions appeared or progressed; neither did she admit self-infliction.

Psychiatric history indicated that she had low mood, loss of interest and enjoyment, reduced concentration, pessimistic views about the future, reduced self-esteem and self-confidence, with fleeting suicidal ideation over the previous six months.

Mental state examination revealed an alert, co-operative female with poor eye contact and a stooped posture. Her speech was slow, softly articulated and monotonous with a delayed response to questions. Her mood was depressed with constricted affect which was decreased in range and reactivity. Her thought content was mood congruent. She had episodic suicidal ideation but had no plans for self-harm. Her cognitive functions were unremarkable.

As her skin lesions did not conform to a recognised dermatological disorder and due to the lesions being present only on more accessible regions of the body, self-inflicted wounds were considered as a possibility. Her history and clinical presentation were also deemed compatible with a diagnosis of a depressive episode.
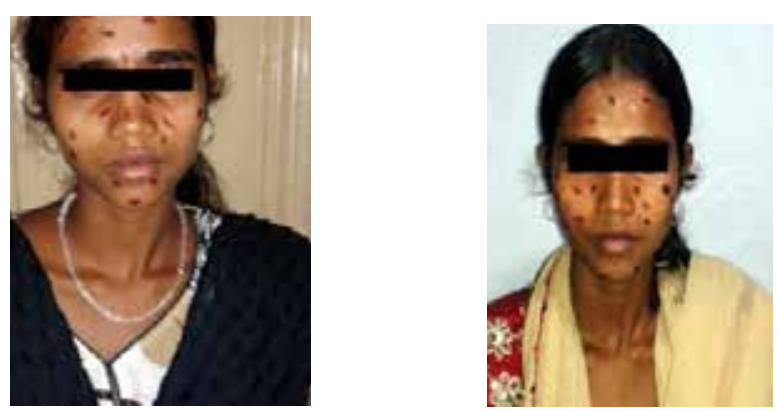
She was prescribed fluoxetine $20 \mathrm{mg}$ daily and offered supportive psychotherapy. After two weeks, the lesions healed with minimal scarring but new lesions were observed (Figure 2). After one month of management with anti-depressants and supportive psychotherapy the lesions ceased to manifest. Thereafter, the patient admitted to self-infliction. Currently, the patient is on anti-depressants and remains well.

\section{Discussion}

Dermatitis artefacta (DA) is a skin disease caused by the deliberate actions of an individual on the skin, hair, nails, or the mucosa. In this disorder, the responsibility for the lesions is uniformly denied by the patient. The diagnosis of such a condition is difficult because of the variability of the skin lesions and the possibility of misdiagnosing a genuine skin lesion.

This psycho-cutaneous disease is classified as a factitious disorder. The Diagnostic and Statistical Manual of Mental Disorders provides the diagnostic criteria of intentional production or feigning of physical or psychological signs or symptoms, the motivation for the behaviour being the need to assume the sick role and external incentives for the behaviour (such as economic gain, avoiding legal responsibility, or improving physical well-being, as in malingering) being absent (1).

Factitious disorders were first described in modern literature by Asher in 1951 (2). The onset is usually during adolescence or in adults under 30 years of age (3). It is commoner in females, the ratio varying 20:1 to $4: 1$, while in children there is equal sex incidence (4). The pathophysiology of DA is poorly understood, but may be multifactorial including the complex interplay between genetic and psychosocial factors (4). The underlying psychopathology is that of assuming a sick role (5).

Usually, no external incentive, economic or legal, is apparent. However, psychosocial stresses in the form of childhood abuse, marital trauma, physical problems, substance abuse, guilt, dysfunctional parent-child relationships or depressive disorder may be associated factors (6).

The most common site of involvement is the face, followed by the dorsum of hands and forearms. The lesions are typically polymorphic, bizarre, clearly demarcated from the surrounding normal skin and can resemble many inflammatory skin reactions. They are usually crude, angulated, and have the tendency for a linear configuration (6).

Characteristically, the patient is unable to provide a clear history of the evolution of the lesions and typically denies any role in the production of the lesions (a 'hollow history'). Lesions evolve overnight without prior signs and symptoms (7).
The management of these patients needs to be nonconfrontational and flexible and involves building a mutual trust and rapport between patient and doctor (7). Clinicians needs to build-up a relationship with the patient through frequent visits and symptomatic treatment and gradually explore the underlying complex personality and behavioural issues associated with the individual.

\section{Declaration of interest}

None

\section{Seshadri Sekhar Chatterjee}

Kaberi Bhattacharya

Department of Psychiatry, Medical College, Kolkata, West

Bengal, India

Corresponding author: Seshadri Sekhar Chatterjee

Email:itismeseshadri@gmail.com

\section{References}

1. American Psychiatric Association. Diagnostic and Statistical Manual of Mental Disorders. DSM-IV, 4 th ed. Text revision. Washington DC: American Psychiatric Press, Inc.; 2000.

2. Asher R. Munchausen's syndrome. Lancet. 1951;1:339341.

3. K. A. Phillips, Somatoform and Factitious Disorders: Review of Psychiatry, vol. 20, 2001.

4. Koblenzer CS. Dermatitis artefacta: Clinical features and approaches to treatment. Am J Clin Dermatol 2000;1:4755.

5. Lyell A. Cutaneous artifactual disease: a review, amplified by personal experience. J Am Acad Dermatol 1979; 1: 391-407.

6. Rogers M, Fairley M, Santhaman R. Artefactual skin disease in children and adolescents. Australas J Dermatol 2001;42:264-70.

7. Verraes-Derancourt S, Derancourt C, Poot F, Heenen M, Bernard P. Dermatitis artefacta: Retrospective study in 31 patients. Ann Dermatol Venereol 2006;133:235-8. 